\title{
Impact of Precision Seeding on Yield Of Sugar Beet
}

\author{
Vasilenko V. V. \\ Voronezh Agrarian University, \\ 394087, Voronezh, Michurin Av., 1, Russia \\ E-mail: vladva.vasilenko@yandex.ru
}

\author{
Vasilenko S. V. \\ Voronezh Agrarian University, \\ 394087, Voronezh, Michurin Av., 1, Russia \\ Voronezh, Russia
}

\author{
Achkasova N N \\ Financial University under the Government \\ of the Russian Federation \\ Moscow, Leningradsky prospect, 49, Russia
}

\begin{abstract}
The yield of sugar beet depends not only on soil and climatic conditions, but also on the quality of the distribution of plants along the sowing series of these plants. The maximum yield is obtained with the optimal value of the average interval between plants, and all intervals should be the same with a coefficient of variation close to zero. A computer program has been computed for the relative yields, depending on the precision of the sowing and the germination of the seeds. The harvest is calculated in relative terms to exclude the influence of soil and weather conditions. For a maximum, the harvest is taken with the exact location of the optimal number of plants under the same germination conditions. The article contains a calculation program and a graphical representation of the results for sugar beet. This will help determine the rational limits of the number of plants per hectare and the accuracy of their distribution. The accuracy of the distribution of plants is estimated by the coefficient of variation of the intervals between them, which can take values from 0.2 to 1.0. The loss of yields from inaccurate distribution of plants along the row may reach $21 \%$.
\end{abstract}

Keywords—sugar beet, seeding, harvest, plants destribution.

\section{INTRODUCTION}

The improvement of the technology of sowing cultivated crops and designs of seeders is aimed at increasing the accuracy of seed distribution, and consequently, of plants along the sowing line. All intervals between plants should be the same, but scientific information is needed on how the accuracy of intervals affects the crop yield of the crop and whether further improve-ment should be continued in this direction. There are a lot of scientific publications on the influ-ence of the number of plants per hectare on crop yields, but detailed information on the joint influence of density and accuracy of plant distribution is proposed for the first time. In the lit-erature, there is evidence of obtaining a good harvest with a plant density of 95-98 thousand plants per hectare $[1,2]$. With a row spacing of $50 \mathrm{~cm}$, a high yield was obtained on a field with a density of planting 10 plants per $\mathrm{m} 2$, that is, 100 thousand plants per hectare [3]. Many studies have shown that the highest yields of sugar beets are reached with plantations of at least 80,000 plants per hectare $[4,5,6]$. In all these studies, it is considered that the plants are located at the same or almost identical intervals from each other. But in fact seeders give an error in accurately laying each seed, not all seeds germinate, and when germinating deviate from the vertical. These factors accumulate inaccuracy in the location of plants, and the more this inaccuracy, the greater the loss of yield. Some authors believe that it is necessary to improve the accuracy of seed placement during sowing [7]. Therefore, the study of the influence of the accuracy of plant placement on crop yield remains relevant.

The proposed method of calculation is based on the application of probability theory to the study of the formation of an interval in a number of plants and numerous measurements of the productivity of each plant, depending on both intervals separating it from neighboring plants. It is assumed that the random deviations $\delta$ of each seed from the calculated location of its placement in the sowing groove are subject to the law of the normal probability density of the distribution. The mathematical expectation $\mathrm{ml}$ of the interval in the row of seeds and the value of $\delta$ determine the coefficient of variation V1. These data are sufficient to obtain the law of probability density of intervals between seeds, known as the gamma distribution. Based on this law, we calculated the probability of pair combinations of two random intervals that separate each seed from both neighbors. Each combination of intervals corresponds to a certain level of plant productivity in relation to the maximum possible productivity with the unlimited feeding area. We obtained this information on the basis of numerous measurements of the productivity of individual plants. The program of computer calculation of productivity from one hectare is made depending on accuracy of distribution of seeds. The program takes into account the fact that not all seeds will germinate, that is, a factor $p$ is introduced that determines a part of the seeds capable of germinating in the field. To avoid the influence of soil and weather factors, the calculation of yields is not in kind, but in relative terms in relation to the maximum possible yield in these soil and weather conditions

\section{METHOD AND CALCULATION MATERIALS}

The calculation is carried out in the operating environment of Mathcad: 
1. Input of initial data characterizing the quality of sowing

$$
m_{1}:=\mathrm{A}, \sigma_{1}:=\mathrm{B}, V_{1}:=\sigma_{1} \cdot m_{1}^{-1}, p:=\mathrm{C},
$$

where $m_{l}$ is the mathematical expectation of the interval between seeds in furrow, $\mathrm{cm}$;

$\sigma_{l}-$ the mean square deviation of the interval, $\mathrm{cm}$;

$V_{l}$ - the variation coefficient of the interval between seeds;

$p$ - field germination of seeds;

A, B - numerical values obtained as a result of statistical data processing in quality control of sowing;

$\mathrm{C}$ - the expected field germination of seeds, expressed in fractions units.

\section{Assignment of boundary values}

$$
x:=2,4 \ldots 150, y:=2,4 \ldots 150, n:=1,2 \ldots 4,
$$

where $x$ and $y$ are the intervals between the seeds in the sowing furrow on either side of each seed; for sugar beet, they can be limited to a size of $150 \mathrm{~cm}$;

$n$ - the number of intervals folded into one, if $\mathrm{n}-1$ seeds, arranged consecutively, did not sprout. The number $n$ can be chosen 3 for a parameter $p$ close to unity, and 6 for $p=0.6 \ldots$ 0.7 .

3. Determination of the law of probability density of the interval between plants on the basis of the gamma distribution of a random variable with its $p$-transformation

$$
\begin{aligned}
& m_{2}:=m_{1} \cdot p^{-1}, q:=1-p, V_{2}:=\sqrt{V_{1}^{2} \cdot p+q}, \alpha:=V_{1}^{-2}, \\
& \beta:=m_{1} \cdot \sigma_{1}^{-2}, f(x):=\sum_{n} \frac{\beta^{n \cdot \alpha}}{\Gamma(n \cdot \alpha)} \cdot x^{n \cdot \alpha-1} \cdot e^{-\beta \cdot x} \cdot p \cdot q^{n-1},
\end{aligned}
$$

where $m_{2}$ is the mathematical expectation of the interval between plants, $\mathrm{cm}$;

$q$ - probability that the seed does not germinate;

$V_{2}$ - coefficient of variation of the interval between plants;

$\alpha, \beta-$ parameters of the gamma distribution;

$x$ - the random value of the interval, $\mathrm{cm}$.

4. Definition of the indicator of intensity of use of the plant nutrition area and average statistical productivity of one plant

$$
\lambda(x, y):=\quad \lambda \max \cdot\left(\frac{0,5 \cdot x+0,5 \cdot y+D}{E}\right)^{2} \cdot\left[1-0,1 \cdot\left(\frac{x-y}{x+y}\right)^{2}\right],
$$

where $\lambda(x, y)$ is the indicator of intensity of use of the plant nutrition area with two random intervals $x$ and $y$ (empirically dependence);

$\lambda \max -$ the indicator of intensity of use of the plant nutrition area of the plant maximal productiveweight growing in free conditions (table 1);

$D, E$ - factors, selected empirically for sugar beet (table 1).
TABLE I. EMPIRICAL COEFFICIENTS FOR THE CALCULATION OF THE INDICATOR INTENSITY OF USE OF THE AREA OF A FEED BY ONE PLANT

\begin{tabular}{|c|c|c|c|c|}
\hline Culture & $\begin{array}{c}\text { Row spacing, } \\
\text { cm }\end{array}$ & $\lambda_{\max }$ & $\boldsymbol{D}$ & $\boldsymbol{E}$ \\
\hline Sugar beet & 45 & 0.110 & 90 & 135 \\
\hline
\end{tabular}

$F(x, y):=1-0,5 \cdot\left[e^{-0,5 \cdot x \cdot \lambda(x, y)}+e^{-0,5 \cdot y \cdot \lambda(x, y)}\right]-0,1 \cdot e^{-0,7 \cdot(x+y) \cdot \lambda(x, y),}$ where $F(x, y)$ is the average productivity of a single plant in fractions of the unit from the maximum possible. This plant is located at intervals $\mathrm{x}$ and $\mathrm{y}$ to its neighbours. The weight $F(x$, $y$ ) of its root is expressed in fractions of unity with respect to the weight of the root crop with intervals $x \rightarrow \infty$ and $y \rightarrow \infty$.

5. Determination of the average relative productivity of the plant throughout the field

$$
g:=\int_{4}^{150150} \int_{4}^{5} f(x) \cdot f(y) \cdot F(x, y) \cdot d x \cdot d y,
$$

where $g$ is the average productivity of a single plant in a given field in fractions of the unit from the maximum possible.

\section{Determination of the relative yield of sugar beet roots on} this field

$$
G:=\frac{g}{m_{2} \cdot G_{\max }},
$$

where $G_{\max }$ is the maximum value of the auxiliary function $G(x)=\frac{F(x)}{x}$, that is, the maximum yield level at the optimal value of all intervals $x$ (Table 2 ).

TABLE II. THE MAXIMUM VALUE OF THE AUXILIARY FUNCTION $G(\mathrm{X})$ AND OPTIMAL INTERVALS BETWEEN PLANTS

\begin{tabular}{|c|c|c|c|}
\hline Culture & Row spacing, $\mathbf{c m}$ & $\boldsymbol{G}_{\mathbf{m a x}}$ & $\boldsymbol{D}$ \\
\hline Sugar beet & 45 & 0,0250 & 20 \\
\hline
\end{tabular}

The program allows you to calculate how an average number of plants is formed per one meter of the row length, depending on the seeding rate and field germination of seeds, what will be the coefficient of variation of the interval between plants and what relative yield level should be expected at this quality of sowing.

\section{RESULTS OF CALCULATION}

For practical use, the plot of dependences of relative yields on two indices - the average number of plants $\left(\mathrm{m}_{2}^{-1}\right)$ per one meter of the row length and the variation coefficient $\left(V_{2}\right)$ of the interval between them is of interest. The yield level is shown in relative units to the maximum possible. The maximum possible level can be achieved with the optimum number of plants per meter of a single seeding row and their absolutely accurate distribution (Figure 1). 


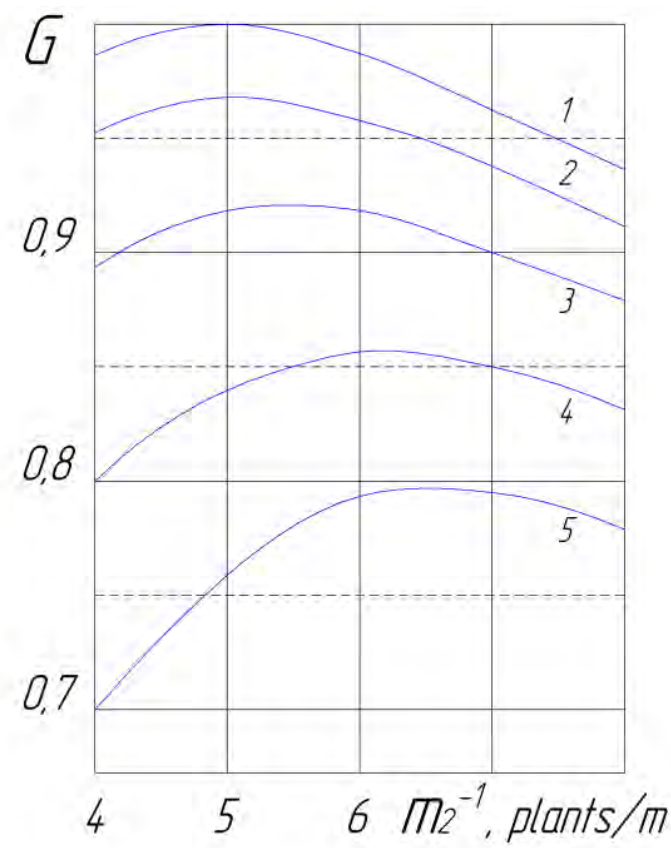

Fig. 1. The graph of the dependence of the relative productivity of sugar beet on the plant population density and the coefficient of variation of intervals between plants if the distance between the rows is $0.45 \mathrm{~m}$

Curve 1 characterizes the most accurate sowing with the use of high-quality seeds with high field germination, as a result of which, at the time of harvesting, the coefficient of variation of inter-vals between plants was small, only $\mathrm{V} 2=0.2$. This curve shows that to achieve the maximum possible yield, it is desirable to have a density of 5 plants per meter, that is 111 thousand plants per hectare. Then the relative yield will come close to unity or to $100 \%$. But if seeding failed to properly place the seeds or they had low germination capacity, then by the time of harvesting the distribution of plants would be chaotic. Chaotic distribution has a coefficient of variation of in-tervals $\mathrm{V} 2=1.0$, and then the best yield is expected at a density of planting 6.5 pieces per meter, that is, 144 thousand pieces per hectare. In this case, we should expect a relative yield of 0.79 or $79 \%$. The loss of productivity of root crops because of poor quality of sowing will be equal to $21 \%$.

As a confirmation of the reliability of the graph in figure 1 , some literature data can be cited. The source [6] states that when the density of plantings changes from 80 to 120 thousand plants per hectare (from 3.6 to 5.4 plants $/ \mathrm{m}$ ), the yield does not fall below $97 \%$. This is in good agreement with curve 1 of our graph. The same authors concluded that the optimal density of sugar beet does not depend on the type of soil. The source [8] reported that with an increase in the density of the plantation to 80 thousand/ha (3.6 plants $/ \mathrm{m})$, the harvest yield significantly increases, but remains almost the same with a further increase in the density of the plantation to 105 thousand/ha ( 4.7 plants $/ \mathrm{m})$. There is evidence that the maximum yield is observed at a plantation density of 90-100 thousand/ha [2]. The most detailed information is given by Minx L [9]. According to him, the maximum yield was obtained at a density of 96.6 thousand / ha (4.4 piec-es / $\mathrm{m}$ ). When the density of the plantation changed from 3.60 to 5.56 pieces $/ \mathrm{m}$, the yield was reduced by $1 \%$.

\section{CONCLUSION}

Thus, the proposed method of calculation makes it possible to determine the indices of plant distribution in relation to the precision of sowing and to calculate the expected relative yield of the crop. The graph of the relative yield of sugar beet root crops shows that the number of plants per one meter of the length of one row from 4.0 to 5.6 has very little effect. For example, sugar beet with a good accuracy of distribution of plants with a coefficient of variation of intervals V2 $=0.2 \ldots 0.4$ shows practically the same yield when the number of plants varies within these lim-its. This means that with a good precision of seeding high-quality seeds, the density of the plantation can be kept within a wide range from 90 to 130 thousand plants per hectare. A much greater influence is exerted by the coefficient of variation of V2. It depends on the quality of sowing and germination of seeds, and with an increase in this indicator, the yield is very signifi-cantly reduced. If the coefficient of variation of the intervals approaches unity, the yield loss will be $21 \%$. The worse the distribution of plants, the more the yield maximum shifts in the graph towards a more dense density of the plantation.

\section{References}

[1] BU. Prosba-Bialczyk, E. Sacala, M. Wilkosz et al, "Impact of seed stimulation and foliar fertiliza-tion with microelements on changes in the chemical composition and productivity of sugar beet", Journal of elementology, 2017, vol. 22, No. 4, pp. 1525-1535.

[2] R. Cakmakci, E. Oral, F. Kantar, "Root yield and quality of sugar beet (Beta vulgaris L.) in rela-tion to plant population" , Journal of agronomy and crop science, 1998, vol. 180, No. 1, pp. 45-52.

[3] M. Ahmad, S. Sedaghatjoo S, A. Westphal, "Reproductive capacity of Heterodera schachtii on Thlaspi arvense, Capsella bursa-pastoris and varying populations of Chenopodium album" Journal of plant diseases and protection, 2016, vol. 123, No, 1, pp. 37-42.

[4] M. Bavorova, N. Stockfisch, H. J. Koch, "Effect of plant density on harvesting quality of sugar-beet", Zuckerindustrie, 2000, vol.125, No, 11, pp. $890-897$.

[5] M. Bavorova, H. J. Koch, "Effect of plant density and lifting speed on harvest losses of sugar beet", Listy cukrovarnicke a repaske, 2002, vol. 118, Np. 9-10, pp. 208-209.

[6] K. W. Jaggard, A. Qi, G. F. J. Milford et al," Determining the optimal population density of sug-arbeet crops in England", International sugar journal, 2011, vol. 1346, No. 113, pp. 114-119.

[7] D. Pochi, R. Fanigliulo," Seed spacing uniformity of 4-6-8 rows precision drills for sugar beet sowing", Acta Horticulturae, 2011, vol. 919, pp. 131138.

[8] I. Tirczka, C. Kondora, "Yield and quality of sugar beet as a function of region, plant density and harvest time", 1999, Novenytermelesm vol. 48, No. 3, pp. 289-299.

[9] L. Minx, Rostlinna Vyroba, 1992, vol. 38, No. 6, pp. 511-515. 